This document is confidential and is proprietary to the American Chemical Society and its authors. Do not copy or disclose without written permission. If you have received this item in error, notify the sender and delete all copies.

\title{
The muscle metabolome differs between healthy and frail older adults
}

\begin{tabular}{|r|l|}
\hline Journal: & Journal of Proteome Research \\
\hline Manuscript ID & pr-2015-00840a.R2 \\
\hline Manuscript Type: & Article \\
\hline Date Submitted by the Author: & 27-Dec-2015 \\
\hline Complete List of Authors: & $\begin{array}{l}\text { Fazelzadeh, Parastoo; Wageningen University, Human Nutrition } \\
\text { Hangelbroek, Roland; Wageningen University, Human Nutrition } \\
\text { Tieland, Michael; Wageningen University, Human Nutrition } \\
\text { de Groot, Lisette; Wageningen University, Human Nutrition } \\
\text { Verdijk, Lex; Maastricht University, Human Movement Sciences } \\
\text { van Loon, Luc; Maastricht University, Human Movement Sciences } \\
\text { Smilde, Age; Swammerdam Institute for Life Sciences, } \\
\text { Alves, Rodrigo; Leiden University, Analytical BioSciences } \\
\text { Vervoort, Jacques; Wageningen University, Laboratory of Biochemistry } \\
\text { Muller, Michael; University of East Anglia, Norwich, Institute of Food } \\
\text { Research } \\
\text { van Duynhoven, John; Wageningen University, Laboratory of Biophysics } \\
\text { Boekschoten, Mark; Wageningen University, Human Nutrition }\end{array}$ \\
\hline
\end{tabular}

\section{SCHOLARONE}

Manuscripts 


\section{The muscle metabolome differs between healthy and frail older adults}

Parastoo Fazelzadeh $^{\S \propto=}$, Roland W.J. Hangelbroek ${ }^{\S \propto=}$, Michael Tieland ${ }^{\S \propto}$, Lisette CPGM de Groot $^{\S \triangleright}$, Lex B Verdijk ${ }^{\S \Delta}$, Luc JC van Loon ${ }^{\S \Delta}$, Age K. Smilde $^{\ddagger}$, Rodrigo DAM Alves ${ }^{ \pm \square}$, Jacques Vervoort $^{\phi}$, Michael Müller ${ }^{+}$, John P.M. van Duynhoven ${ }^{\square r^{*},}$ Mark V Boekschoten ${ }^{\S 凶}$

${ }^{\S}$ Top Institute Food and Nutrition, Wageningen, The Netherlands

Nutrition, Metabolism and Genomics group, Division of Human Nutrition, Wageningen University, Wageningen, The Netherlands

${ }^{\Delta}$ Department of Human Movement Sciences, NUTRIM School of Nutrition and Translational Research in Metabolism, Maastricht University, Maastricht, the Netherlands

${ }^{\ddagger}$ Biosystems Data Analysis group, University of Amsterdam, Amsterdam, The Netherlands

${ }^{£}$ Department of Analytical Biosciences, Leiden University, Leiden, The Netherlands

${ }^{\phi}$ Laboratory of Biochemistry, Wageningen University, Wageningen, The Netherlands

'Haboratory of Biophysics, Wageningen University, Wageningen, The Netherlands

${ }^{+}$Norwich Medical School, University of East Anglia, United Kingdom

${ }^{\square}$ Netherlands Metabolomics Centre, Leiden, The Netherlands

= These authors contributed equally to this study. 


\begin{abstract}
:
Populations around the world are aging rapidly. Age-related loss of physiological functions negatively affects quality of life. A major contributor to the frailty syndrome of aging is loss of skeletal muscle. In this study we assessed the skeletal muscle biopsy metabolome of healthy young, healthy older and frail older subjects to determine the effect of age and frailty on the metabolic signature of skeletal muscle tissue. In addition, the effects of prolonged whole-body resistance-type exercise training on the muscle metabolome of older subjects were examined. The baseline metabolome was measured in muscle biopsies collected from 30 young, 66 healthy older subjects and 43 frail older subjects. Follow-up samples from frail older (24 samples) and healthy older subjects (38 samples) were collected after 6 months of prolonged resistance-type exercise training. Young subjects were included as a reference group. Primary differences in skeletal muscle metabolite levels between young and healthy older subjects were related to mitochondrial function, muscle fiber type, and tissue turnover. Similar differences were observed when comparing frail older subjects with healthy older subjects at baseline. Prolonged resistance-type exercise training resulted in an adaptive response of amino acid metabolism, especially reflected in branched chain amino acids and genes related to tissue remodeling. The effect of exercise training on branched-chain amino acid-derived acylcarnitines in older subjects points to a downward shift in branched-chain amino acid catabolism upon training. We observed only modest correlations between muscle and plasma metabolite levels, which pleads against the use of plasma metabolites as a direct read-out of muscle metabolism and stresses the need for direct assessment of metabolites in muscle tissue biopsies.
\end{abstract}

KEYWORDS: muscle biopsy, frailty, aging, tissue remodeling 


\section{INTRODUCTION}

Populations around the world are aging rapidly. It has been estimated that people older than 60 years of age will make up $22 \%$ of the world population, whereas people older than 80 years of age will account for $4.4 \%$ of the world population in $2050{ }^{1}$. Age-related loss of physiological functions compromises independence at older age. A major contributor to the frailty syndrome of aging is skeletal muscle loss, which can lead to increased disability in the older population. In most people muscle mass and strength start to decline around the age of 35 with more progressive muscle loss observed after the age of $65^{2-6}$.

Exercise training has been applied to counter the effects muscle mass loss, such as loss of strength 7,8 . We recently demonstrated that prolonged resistance-type exercise training partially shifts the skeletal muscle transcriptome of older subjects toward an expression pattern observed in muscle tissue of young subjects, with changes in gene expression related to vascularisation, tissue remodelling and glucose metabolism ${ }^{9}$. The transcriptome analysis also revealed substantial differences between healthy young men, healthy older subjects and frail older subjects before any intervention was undertaken. In particular, genes related to mitochondrial function had lower expression levels in older subjects compared to young subjects.

The age-related differences in gene expression level in muscle are expected to be reflected in local levels of metabolites. However, limited information is available on the effect of aging on muscle metabolite levels. A recent study in mice showed that aging affects muscle glucose and fatty acid metabolism ${ }^{10}$, whereas a study in rats indicated that aging leads to muscle group-specific perturbations in lipid and glucose metabolism consistent with mitochondrial dysfunction ${ }^{11}$. In humans, it was found that changes in lipid content and oxidative activity in skeletal muscle during aging are related to a shift in muscle fiber type ${ }^{12}$. Interestingly, exercise training led to reprogramming of mitochondrial function and intermediary 
metabolism in insulin-insensitive obese subjects ${ }^{13}$. In both human studies, the metabolic profiling platforms that were used had limited coverage and focused on specific submetabolomes. A major bottleneck in achieving extended coverage has been the limited amount of muscle tissue material that can be sampled from human subjects.

On the basis of the observed shift in transcriptome profiles after training we hypothesized that a similar shift towards the young phenotype occurs in the muscle metabolome after resistancetype exercise training in older subjects. The recent development of a comprehensive targeted metabolic profiling platform optimized and validated for small muscle biopsies makes it possible to elaborately verify this hypothesis ${ }^{14}$. Here we aim to investigate the effect of aging and frailty on the skeletal muscle metabolome. In addition, we examined the impact of prolonged resistance-type exercise training on the metabolome of frail and healthy older subjects.

\section{MATERIALS AND METHODS}

\section{Experimental design}

Muscle biopsies ( $m$. vastus lateralis), plasma and serum samples were collected from frail older $^{15,16}$, healthy older ${ }^{17,18}$ and young subjects ${ }^{19}$. The frail group included both frail and pre-frail subjects and are hereafter referred to as frail. Medical history of all subjects was evaluated by medical questionnaires, which were analyzed by a physician. Subjects who were unable to participate in the exercise program due to pain were excluded prior to starting the intervention. Included subjects who showed severe discomfort during the training sessions were excluded from further participation in the study. Baseline metabolite levels were measured in skeletal muscle tissue of 30 young, 66 healthy and 43 frail older subjects (Table 1). We also measured metabolites in plasma and serum for 50 young, 76 healthy and 62 frail 
older subjects (Supporting Information Table S1). The transcriptome of the muscle samples was measured in an earlier study ${ }^{9}$.

Baseline samples from healthy young male subjects served as reference and were derived from several studies performed within our group, in which exactly the same techniques and processing were used for sample collection. Samples from the frail and healthy older subjects were obtained from two study centers. More details of the studies can be found in the papers published previously ${ }^{15,18}$. In addition, muscle biopsies were obtained after 6 months of resistance-type exercise training for 38 healthy and 24 frail older subjects. All muscle biopsies and blood samples were obtained in the morning after an overnight fast. Subjects ate a standardized meal the evening before sample collection. Subjects refrained from doing any strenuous physical activity for 3 days prior to muscle biopsy collection.

Fried criteria ${ }^{20}$ were used to assess the frailty in older subjects, which takes into account unintentional weight loss, weakness, self-reported exhaustion, slow walking speed, and low physical activity. Based on the above mentioned criteria, the healthy older subjects were not considered frail or pre-frail at the start of the intervention study ${ }^{21}$.

In our study population, all older subjects regardless of their health status (frail or healthy) improved in muscle performance following 6 months of resistance-type exercise training (Supporting Information Figure S1 Table S2 ), as illustrated by significantly increased leg extension and leg press strength after training $(P \text {-value }<0.01)^{9,15,21}$. Both healthy older and frail older subjects followed similar progressive full-body resistance type exercise training. In brief, the training consisted of a 5 minute warm-up on a cycle ergometer, followed by 4 sets on the leg-press and leg-extension machines. In addition, 3 sets of chest press, lat pulldown, pec-dec and vertical row machines were performed (Technogym, Rotterdam, The Netherlands). The healthy older subjects trained 3 times per week and frail subjects trained 2 times per week. Moreover, subjects received a protein or control supplement during the study. 
Healthy older subjects took 15 gram milk protein or control drink at breakfast and frail older subjects took a similar 15 gram drink (milk protein or control) at breakfast and lunch every day throughout the entire 6 months intervention. Full details can be found in the earlier papers on the phenotypical impact of training on our study population ${ }^{15,21}$. All studies were approved by The Medical Ethics Committee of either Wageningen University or Maastricht University and comply with the Declaration of Helsinki.

\section{Metabolomics analysis of circulating metabolites}

Amino acids and biogenic amines were derivatized (Acc-Tag) in $5 \mu \mathrm{L}$ aliquots of plasma. Samples were analyzed using an ACQUITY UPLC system with autosampler (Waters, EttenLeur, The Netherlands) coupled with a Xevo Tandem quadrupole mass spectrometer (Waters) operated using QuanLynx data acquisition software (version 4.1; Waters). An Accq-Tag Ultra column (Waters) was used. The Xevo TQ was used in the positive-ion electrospray mode and all analytes were monitored in Multiple Reaction Monitoring (MRM) using nominal mass resolution. Acquired data were evaluated using TargetLynx software (Waters), by integration of assigned MRM peaks and normalization using proper internal standards ${ }^{22}$,

Acylcarnitines, trimethylamine-N-oxide, choline, betaine, deoxycarnitine and carnitine were analyzed in $5 \mu \mathrm{L}$ plasma, spiked with an internal standard, using a UPLC-MS/MS. Also here an Accq-Tag Ultra column was used. The Xevo TQ was used in the positive-ion electrospray mode and all analytes were monitored in MRM using nominal mass resolution. In-house developed algorithms ${ }^{23}$ were applied using the pooled QC samples to compensate for shifts in the sensitivity of the mass spectrometer over the batch.

Organic acids were measured by GC-MS using $50 \mu \mathrm{L}$ of plasma sample prepared using a twostep derivatization procedure with subsequent oximation using methoxyamine hydrochloride (MeOX) and silylation using N-Methyl-N-(trimethylsilyl)trifluoroacetamide (MSTFA. Samples were measured on an Agilent GC (7890A) coupled to Agilent Quadrupole-MS with 
EI source (Agilent MSD 5975C). Separation was performed using a HP-5MS column (30 m x $0.25 \mathrm{~m} \times 0.25 \mu \mathrm{m}$; Agilent). The raw data were pre-processed using Agilent MassHunter Quantitative Analysis software for GC-MS (Agilent, Version B.04.00), and quantitation of metabolite response was calculated as the peak area ratios of the target analyte to the respective internal standard. In-house developed algorithms were applied using the pooled QC samples to compensate for shifts in the sensitivity of the mass spectrometer over the batch. Serum metabolite concentrations determined by NMR were measured as described by Mihaleva et al ${ }^{24}$. In short, serum samples were ultrafiltrated and automated quantum mechanical line shape fitting of ${ }^{1} \mathrm{H}$ NMR spectra was performed using PERCH.

\section{In tissue metabolome analysis}

Metabolites were extracted from $10 \mathrm{mg}$ of wet muscle tissue. This tissue was further lyophilized and weighted to determine the dry tissue mass. After pulverizing the tissue, metabolites were extracted using methanol/chloroform/water (MCW). The extraction method used in this study has extensively been described and characterized elsewhere ${ }^{14}$.

Amines, acylcarnitines and oxylipins were measured using the platforms also used for measurement of these metabolites in plasma. The validation of these methods for human tissue biopsies is described in detail elsewhere ${ }^{23}$. In-house developed algorithms were applied to compensate for shifts in the sensitivity of the mass spectrometer over multiple batches of measurements ${ }^{23}$. The metabolite response was determined by the peak area ratio of the target analyte to the appropriate internal standard. These response ratios were used in the subsequent data analysis. ATP, ADP, creatine and phosphocreatine were determined using established enzymatic assays. For the first 3 metabolites, commercially available fluorimetric assay kits were used (BioVision cat.\# K354-100, K355-100 and K635-100) following the manufacturer's instructions. Phosphocreatine was measured according to a colorimetric assay 
kit protocol described by Szas et al. ${ }^{25}$. In total 96 metabolites including amine, acylcarnitines, organic acids, oxylipins and a number of nucleotides were measured.

\section{Statistical analysis}

Statistical analysis was performed on log-transformed data. We used analysis of variance (ANOVA) for between group comparisons at baseline. $P$-value $<0.05$ was considered statistically significant. We used linear mixed models for assessment of the training effect. Our model included exercise training, subject, sex, protein supplementation and within subject correlation. Analyses of the training effect was performed separately for frail and healthy older subjects due to differences in training frequency. To summarize acylcarnitines into one single metabolite (eigenmetabolite), the Non-linear Iterative partial least squares (NIPALS) algorithm ${ }^{26}$ of the mixOmics R library was used to calculate the Singular Value Decomposition (SVD) of acylcarnitines. To integrate metabolomics and transcriptomics data the multilevel sparse partial least squares (sPLS) module from the mixOmics R library was used $^{27}$. We applied canonical correlation ( cut-off $=>0.8$ ), which highlights the strongest correlations between the two data sets. FactoMineR was used to perform principal component analysis (PCA) ${ }^{28}$. All analyses were done using R (version 3.02).

ASCA (ANOVA Simultaneous Component Analysis) was performed to determine global differences on metabolites. ASCA is a multivariate method that partitions variation in the data and enables to interpret these partitions by simultaneous component analysis ${ }^{29}$. Analysis was performed separately for the frail and healthy older subjects, using delta values of each metabolite for each individual (value after training - value before training) with supplement, sex and their interaction in the model as factors. Analysis was done under Matlab (version R2012a). 


\section{RESULTS}

\section{Baseline comparisons between healthy older, frail older and young subjects}

Comparison of the plasma metabolite profiles of young and healthy older subjects showed marked differences in levels of many metabolites between the groups. Analysis of variance (ANOVA) showed that differences in plasma metabolite levels between older and young subjects were mainly accounted for by the amino acids and acylcarnitines. These differences were in line with previous observations of age-related effects on the plasma metabolome ${ }^{10,30 \text {, }}$ 31.

Figure 1 shows a principal component analysis (PCA) plot of the biogenic amine profiles of the muscle biopsies obtained from young, healthy older and frail older subjects, before and after exercise. We observed clear age-related differences in the biogenic amine profiles of skeletal muscle, as well as a difference between healthy and frail older subjects. The PCA plot also revealed an effect of prolonged resistance-type exercise training on amine concentrations in muscle tissue. PCA revealed a similar effect for organic acids in muscle (Supporting Information Figure S2).

ANOVA yielded a series of skeletal muscle metabolites that differ significantly between healthy older subjects and young subjects. Many of these metabolites are amino acids and organic acids (Table 2). The outcome of ANOVA modeling of muscle metabolites in healthy vs. frail older subjects is presented in Table 3.

\section{Effect of prolonged resistance-type exercise training in frail and healthy older subjects}

In the PCA plot in Figure 1 we can observe that prolonged resistance-type exercise training has an effect on the biogenic amine profile of skeletal muscle tissue of frail and healthy older subjects. The trends in the PCA plot suggest that upon training both healthy and frail older subjects shift towards a younger phenotype (see also the plot for the average value of isoleucine in Supporting information Figure S3,a). The variation in this PCA plot may not 
only be due to phenotype (young, healthy and frail old) and exercise, but also to sex and protein supplementation. Accordingly, we performed ANOVA Simultaneous Component Analysis (ASCA) to account for these different sources of biological variation. We observed that prolonged resistance-type exercise training had a weak effect on muscle tissue metabolite levels. No significant interaction of protein supplementation with training in both frail and healthy older subjects could be observed. Interaction of sex with training was not significant in healthy older subjects, but was significant in frail older subjects ( $P$-value $=0.03)$.

Next we used linear mixed models on univariate metabolite levels to account for phenotype, exercise, protein supplementation and sex as sources of biological variation. We observed that prolonged resistance-type exercise training affected muscle levels of acylcarnitines in both the healthy older and frail older subjects (Table 4 and 5, respectively). These include acylcarnitines that are produced during oxidation of branched chain amino acids (propionyl (C3), methylmalonyl (C4-DC), and isovaleryl (C5) acylcarnitines) and acylcarnitines that are produced during fatty acid oxidation (C6-C20 acylcarnitines) ${ }^{32}$. Because the exercise effect on several acylcarnitines was nearly significant $(P$-value $>0.05)$, we used the singular value decomposition to summarize the levels of fatty acid derived and amino acid derived acylcarnitines (denoted as FAAC and AAAC, respectively in Figure 4 and 5). Subsequently, we also performed linear mixed model analysis to determine the effect of training on the fatty acid derived and amino acid derived acylcarnitines. The amino acid derived acylcarnitines levels were significantly decreased after training in both healthy and frail older subjects (see example of the effects for propionylcarnitine in Supporting information Figure S3,b).

We used multilevel sPLS to integrate changes in the muscle transcriptome and muscle metabolome after training. Here the goal was to investigate the interaction of two matched datasets and the selection of subsets of either positively or negatively correlated variables across all subjects. Only those genes were selected that were significantly changed by training 
$($ FDR $<0.05)$ in both frail and healthy older subjects. The resulting correlation network in Figure 2 shows that especially branched chain amino acids correlate with genes related to connective tissue/extracellular matrix such as collagen, laminin and Secreted Protein, Acidic, Cysteine-Rich (SPARC) .

\section{Correlation between muscle and plasma metabolites levels}

Next we investigated to what extent baseline plasma metabolite levels reflect muscle metabolite levels. To that end we first constructed separate correlation heatmaps for muscle and plasma metabolites within each compartment. Figure $3 \mathrm{a}-\mathrm{b}$ shows that metabolites that belong to the same group of metabolites (e.g. amino acids, acylcarnitines) are correlated to each other, which is observed for both plasma and muscle. However, the heatmap of the correlation between muscle and plasma metabolites (Figure 3c) showed only minor to moderate correlations (Pearson correlation between 0.3 and 0.5 ). The correlation networks (Figure 3d) revealed the strongest correlations for 3-hydroxybutyric acid, 4-hydroxyproline, proline, branched chain amino acids and several acylcarnitines. Correlations between serum and muscle metabolites are presented in Supporting Information Figure S4. The results are generally similar to the correlations observed between plasma and muscle metabolites.

\section{DISCUSSION}

\section{Comprehensive metabolic profiling of muscle biopsies: differences with age and effect of resistance-type exercise training in a heterogeneous study population}

Comprehensive metabolic profiling of muscle biopsies revealed pronounced differences between the muscle biopsy metabolomes of healthy young, healthy older and frail older subjects. Furthermore, distinct effects of prolonged resistance-type exercise training could be observed in both groups of older subjects.. 
A key asset of our analysis was the deployment of five robust profiling platforms using a single and thoroughly validated muscle-biopsy extraction procedure ${ }^{14}$. The analytical variation in these profiles was around $20-30 \%$, which was smaller than the biological variation between the muscle biopsies. In order to separate the sources of biological variation in our heterogeneous study population, we used univariate linear mixed models. Interestingly, the metabolic effects of supplementation and differences between males and females were relatively minor compared to the differences between old and young and between healthy and frail. The effects were also minor compared to the response to prolonged resistance-type exercise training. A multivariate approach (ASCA) did not show any significant effects of training, probably because not all sources of biological variation could be adequately accounted for ${ }^{33}$. In the following discussion we focus on the univariate linear mixed model approach since it allows us to more precisely account for protein supplementation and sex in our study population.

\section{Baseline comparisons}

Interestingly, in muscle biopsies baseline levels of metabolites from the tricarboxylic acid cycle (succinic acid, fumaric acid, 2-ketoglutaric acid) were lower in the healthy older subjects compared to young subjects. These differences were accompanied by lower levels of ATP, ADP, branched chain amino acids and acylcarnitines in the healthy older subjects, suggesting impaired mitochondrial function or a lower number of mitochondria in the muscle of the older subjects, which in turn may be a consequence of the lower habitual physical activity of the older subjects. These observations are in line with data on the muscle transcriptomic profiles of these subjects, which show decreased expression of genes related to mitochondrial function and oxidative phosphorylation in the older subjects compared to young subjects, with the lowest expression observed in the frail older subjects ${ }^{9}$. These 
findings are in agreement with other studies ${ }^{10,34-38}$, which also showed a down-regulation of the mitochondrial electron-transport chain pathway in older as compared to young subjects.

One of our findings is that levels of 4-hydroxyproline and proline are lower in the healthy older subjects compared to the young subjects. Both of these amino acids have been associated with collagen turnover ${ }^{39,40}$. Muscle levels of two precursors of proline, ornithine and arginine, are higher in the older subjects, which together with lower levels of 4hydroxyproline and proline may be due to dysfunction of the mitochondrial ornithine aminotransferase ${ }^{41}$, leading to accumulation of arginine and ornithine. This notion is in line with slightly lower expression of genes related to tissue remodeling, such as collagen in the older subjects compared to the young subjects ${ }^{9}$.

We observed higher levels of $\beta$-isoamino butyric acid (BAIBA) in muscle tissue of healthy older subjects compared to young subjects. This metabolite has been suggested to be produced upon exercise by expression of PGC-1 $\alpha$ and has been proposed as a myokine stimulating browning of white adipose tissue and hepatic $\beta$-oxidation ${ }^{42}$. In our study population we observed lower expression of PGC-1 $\alpha$ target genes in healthy older subjects compared to young subjects, and in frail older subjects compared to healthy older subjects ${ }^{9}$. The activity of PGC- $1 \alpha$ is modulated post-translationally by $\mathrm{NAD}^{+}$dependent deacetyltation by SIRT1 (Figure 4-b) ${ }^{43}$. Since NAD $^{+}$levels decrease with age ${ }^{44}$, we expect a concurrent downregulation of PGC-1 $\alpha^{45}$. However, lower levels of BAIBA were only observed when comparing frail to healthy older subjects, whereas BAIBA levels were higher in healthy older subjects compared to young subjects. Assuming that aging lead to down-regulation of PGC$1 \alpha$, the observed changes in baseline muscle BAIBA levels are not consistent with BAIBA serving as a marker for PGC-1 $\alpha$ activity. Thus, we cannot confirm the notion that BAIBA acts as a PGC-1 $\alpha$ induced myokine. A possible explanation for the discrepancy is that in our study 
differences in PGC-1 $\alpha$ expression are caused by age and frailty-dependent processes, whereas previously described PGC- $1 \alpha$-mediated effects on BAIBA were caused by acute exercise.

Two polyamines, spermine and spermidine, were found to be significantly different between the frail in comparison to the healthy older subjects. Polyamines are involved in tissue regeneration and cell proliferation and differences are associated with both exercise and muscle pathology ${ }^{46-48}$. The genes directly involved in the polyamine pathway are however not differentially expressed between frail and healthy older subjects. Hence the observed differences in polyamine levels between the frail and healthy older subjects are more likely to be attributed to effects at the level of enzyme activity or metabolite transport. Previous studies have shown that perturbations in polyamine metabolism are associated with neuromuscular disorders ${ }^{49,50}$. On the transcriptome level we indeed found indications of neuromuscular perturbations in the frail older subjects ${ }^{9}$. In addition, increased spermine levels were recently linked to skeletal muscle atrophy ${ }^{51}$. However, we observed lower levels of spermine in frail subjects in comparison to healthy older subjects, even though frail older subjects generally have less skeletal muscle and are likely to exhibit more extensive muscle atrophy. Ost et al. recently reported that spermidine is increased in the skeletal muscle of mice overexpressing uncoupling protein 1 . The authors proposed that this might be an adaptive response to cope with the additional oxidative stress ${ }^{52}$. This would suggest that also in the frail elderly the increase in spermidine is a response to reduced oxidative capacity and increase in oxidative stress.

The level of carnosine is decreased in healthy older subjects in comparison to young subjects, and in frail in comparison to healthy older subjects. Carnosine is an abundant metabolite in muscle where it plays an important role in intracellular $\mathrm{pH}$ buffering ${ }^{53}$. Carnosine has also been associated with chelation of metal ions and antioxidant activity ${ }^{54}$. Furthermore, carnosine levels are higher in type II muscle fibers compared to type I. A likely explanation 
for the significantly lower levels of carnosine in healthy and frail older subjects is therefore a decrease of the muscle fiber II/I ratio with respectively age and lack of exercise ${ }^{55}$.

Several oxylipins derived from linoleic acid (LA) and $\alpha$-linoleic acid (ALA) occur at higher levels in the muscle of the healthy older subjects compared to young subjects. On the other hand, levels of metabolites derived from the $\Delta-6$ desaturase product dihomo- $\gamma$-linoleic acid (DGLA) are lower in healthy older subjects. We postulate that due to reduced $\Delta-6$ desaturase activity linoleic acid and $\alpha$-linoleic acid accumulate in the muscle of the healthy older subjects, whereas downstream $\Delta-6$ desaturase product dihomo- $\gamma$-linoleic acid products are depleted $^{56}$

\section{Effect of prolonged resistance-type exercise training}

We compared the effect of prolonged resistance-type exercise training in both healthy and frail older subjects on all measured metabolites using multilevel sPLS. There was a profound correlation between the adaptive response to training between the transcriptome and amino acids in the muscle metabolome (canonical correlations between 0.7 and 0.8 ). Particularly high correlations were observed between expression changes of extracellular matrix genes and amino acids. Although it is unlikely that there is a direct link between expression of these genes and levels of these metabolites, it does imply that these changes in amino acid levels are part of the adaptive response to resistance-type exercise training.

At the metabolite level, the most striking effects of resistance-type exercise training in frail and healthy older subjects were observed for the C3 (propionyl) and C5 (isovaleryl) muscle acylcarnitines which are derived from branched chain amino acids. After training, the amino acid derived acylcarnitines showed a significant decrease both in the healthy and frail older subjects, accompanied by an increase of branched chain amino acids. A likely explanation is that the flux-determining mitochondrial branched chain $\alpha$-keto acid hydrogenase (BCKDH) 
complex ${ }^{57}$ has a compromised response to prolonged resistance-type exercise training. As is schematically depicted in Figure 4-a, the BCKDH complex can respond to exercise via different mechanisms. PGC- $1 \alpha$ is a known activator of $\mathrm{BCKDH}$, but training did not have an effect on its gene expression in our study. Exercise is known to increase NAD $^{+}$levels ${ }^{45}$ and could thus activate PGC-1 $\alpha$ in a post-translational manner via SIRT1. Apparently this mechanism is also not activated by training in the older subjects. These effects are specific for branched chain amino acids oxidation and no significant effects on fatty acid derived acylcarnitines were found. A decrease in branched chain amino acids oxidation may stimulate mTOR related pathways activation and protein synthesis or attenuate muscle protein breakdown $^{58,59}$, which could be beneficial for older subjects. As this mechanism occurs at enzymatic level, the available metabolomics and transcriptomics data can however not confirm this hypothesis and in future studies proteomics analysis would be called for.

\section{Correlation of plasma and muscle metabolome}

The weak correlations between plasma and muscle metabolite levels indicate that plasma levels only partially reflect muscle metabolism, even though skeletal muscle is one of the largest metabolically active tissues in the human body. This seems to suggest that these metabolites are also produced by other metabolic compartments. In a recent study, lack of correlation between acylcarnitine levels in plasma and tissues in mice was attributed to differences in turnover in plasma and muscle compartments, and contribution of other compartments than muscle to plasma acylcarnitine levels ${ }^{60}$. The same rationale very likely also applies to amino acids involved in collagen metabolism (proline, 4-hydroxyproline), which can also be formed in bone. Adipose tissue is also a metabolically active compartment with regard to branched chain amino acids besides muscle ${ }^{61}$, and this will likely weaken plasma-muscle level correlations. As a consequence, correlations between plasma metabolites 
and muscle metabolites are too modest to support their use as direct read-outs of muscle metabolism ${ }^{62,63}$.

\section{Conclusion}

The major differences in muscle metabolome of healthy older and young subjects relate to mitochondrial function, fiber-type composition, and tissue turnover. Similar differences were observed when comparing frail older subjects to healthy older subjects. Prolonged resistancetype exercise training resulted in a correlative adaptive response of amino acids and genes responsible for tissue remodeling. The effect of exercise on amino acid derived acylcarnitines in healthy and frail older subjects points towards decreased branched chain amino acids catabolism, likely due to attenuated activation of the flux-determining mitochondrial branched chain $\alpha$-keto acid hydrogenase complex in older subjects. Only modest correlations between muscle metabolite and plasma levels were found, which prohibits the use of the latter as readouts of muscle metabolism.

\section{AUTHOR INFORMATION}

\section{Corresponding author}

*E mail: john.vanduynhoven@,wur.nl. Phone: +31(0)317 482044.

\section{Acknowledgements}

This research was financially supported by Top Institute Food and Nutrition, Wageningen, The Netherlands and the Netherland Metabolomics Centre (NMC), part of the Netherlands Genomics Initiative (NGI). TI Food and Nutrition is a public-private partnership on precompetitive research in food and nutrition. The public partners are responsible for the 
study design, data collection and analysis, decision to publish, and preparation of the manuscript. The private partners have contributed to the project through regular discussion. The authors gratefully acknowledge the staff of the NMC Demonstration and Competency Centre for performing the expert metabolic profiling work on plasma and muscle biopsies.

We also thank Dr.Velitchka V. Mihaleva for expert deconvolution of ${ }^{1} \mathrm{H}$ NMR spectra of serum.

\section{ASSOCIATED CONTENT \\ Supporting Information}

Figure S1. Change of leg extension 1RM after resistance type exercise training. Figure S2. PCA plot of metabolites detected by the organic acid platform in muscle tissue. Figure S3. Group means with 95 percent confidence intervals for isoleucine and propionylcarnitine. Figure S4. Pearson correlation heatmap between muscle and serum metabolites. Table S1. Characteristics of subjects of which plasma samples were studied. Table S2. Characteristics of the older subjects of which samples were obtained before and after training. 


\section{References}

1. PD, U. N., Department of Economic and Social Affairs.Population Ageing and Development .New York. September 2012.

2. $\quad$ Frontera, W. R.; Hughes, V. A.; Fielding, R. A.; Fiatarone, M. A.; Evans, W. J.; Roubenoff, R., Aging of skeletal muscle: a 12-yr longitudinal study. 2000; Vol. 88, p 13211326.

3. Goodpaster, B. H.; Park, S. W.; Harris, T. B.; Kritchevsky, S. B.; Nevitt, M.; Schwartz, A. V.; Simonsick, E. M.; Tylavsky, F. A.; Visser, M.; Newman, A. B., The loss of skeletal muscle strength, mass, and quality in older adults: the health, aging and body composition study. J Gerontol A Biol Sci Med Sci 2006, 61, (10), 1059-64.

4. $\quad$ Sayer, A. A.; Robinson, S. M.; Patel, H. P.; Shavlakadze, T.; Cooper, C.; Grounds, M. D., New horizons in the pathogenesis, diagnosis and management of sarcopenia. Age Ageing 2013, 42, (2), 145-50.

5. Sayer, A. A.; Syddall, H.; Martin, H.; Patel, H.; Baylis, D.; Cooper, C., The developmental origins of sarcopenia. J Nutr Health Aging 2008, 12, (7), 427-32.

6. Sayer, A. A.; Syddall, H. E.; Martin, H. J.; Dennison, E. M.; Anderson, F. H.; Cooper, C., Falls, sarcopenia, and growth in early life: findings from the Hertfordshire cohort study. Am J Epidemiol 2006, 164, (7), 665-71.

7. Snijders, T.; Verdijk, L. B.; van Loon, L. J., The impact of sarcopenia and exercise training on skeletal muscle satellite cells. Ageing Res Rev 2009, 8, (4), 328-38.

8. Morley, J. E., Editorial: Sarcopenia Revisited. The Journals of Gerontology Series A: Biological Sciences and Medical Sciences 2003, 58, (10), M909-M910.

9. Hangelbroek, R. W. J.; Fazelzadeh, P.; Tieland, M.; Boekschoten, M. V.; Hooiveld, G. J. E. J.; van Duynhoven, J. P. M.; Timmons, J. A.; Verdijk, L. B.; van Loon, L. J. C.; de Groot, L. C. P. G. M.; Müller, M., Expression of protocadherin gamma in skeletal muscle is associated with muscle weakness and age. In press 2015.

10. Houtkooper, R. H.; Argmann, C.; Houten, S. M.; Canto, C.; Jeninga, E. H.; Andreux, P. A.; Thomas, C.; Doenlen, R.; Schoonjans, K.; Auwerx, J., The metabolic footprint of aging in mice. Sci Rep 2011, 1, 134.

11. Garvey, S. M.; Dugle, J. E.; Kennedy, A. D.; McDunn, J. E.; Kline, W.; Guo, L.; Guttridge, D. C.; Pereira, S. L.; Edens, N. K., Metabolomic profiling reveals severe skeletal muscle group-specific perturbations of metabolism in aged FBN rats. Biogerontology 2014, $15,(3), 217-32$.

12. Gueugneau, M.; Coudy-Gandilhon, C.; Théron, L.; Meunier, B.; Barboiron, C.; Combaret, L.; Taillandier, D.; Polge, C.; Attaix, D.; Picard, B.; Verney, J.; Roche, F.; Féasson, L.; Barthélémy, J.-C.; Béchet, D., Skeletal Muscle Lipid Content and Oxidative Activity in Relation to Muscle Fiber Type in Aging and Metabolic Syndrome. The Journals of Gerontology Series A: Biological Sciences and Medical Sciences 2014.

13. Huffman, K. M.; Koves, T. R.; Hubal, M. J.; Abouassi, H.; Beri, N.; Bateman, L. A.; Stevens, R. D.; Ilkayeva, O. R.; Hoffman, E. P.; Muoio, D. M.; Kraus, W. E., Metabolite signatures of exercise training in human skeletal muscle relate to mitochondrial remodelling and cardiometabolic fitness. Diabetologia 2014, 57, (11), 2282-95.

14. Alves, R. A. M.; Dane, A.; Harms, A.; Strassburg, K.; Seifar, R.; Verdijk, L.; Kersten, S.; Berger, R.; Hankemeier, T.; Vreeken, R., Global profiling of the muscle metabolome: method optimization, validation and application to determine exercise-induced metabolic effects. Metabolomics 2015, 11, (2), 271-285.

15. Tieland, M.; Dirks, M. L.; van der Zwaluw, N.; Verdijk, L. B.; van de Rest, O.; de Groot, L. C.; van Loon, L. J., Protein supplementation increases muscle mass gain during 
prolonged resistance-type exercise training in frail elderly people: a randomized, doubleblind, placebo-controlled trial. J Am Med Dir Assoc 2012, 13, (8), 713-9.

16. Tieland, M.; van de Rest, O.; Dirks, M. L.; van der Zwaluw, N.; Mensink, M.; van Loon, L. J.; de Groot, L. C., Protein supplementation improves physical performance in frail elderly people: a randomized, double-blind, placebo-controlled trial. J Am Med Dir Assoc 2012, 13, (8), 720-6.

17. Wall, B. T.; Hamer, H. M.; de Lange, A.; Kiskini, A.; Groen, B. B.; Senden, J. M.; Gijsen, A. P.; Verdijk, L. B.; van Loon, L. J., Leucine co-ingestion improves post-prandial muscle protein accretion in elderly men. Clin Nutr 2013, 32, (3), 412-9.

18. Leenders, M.; Verdijk, L. B.; van der Hoeven, L.; van Kranenburg, J.; Nilwik, R.; van Loon, L. J., Elderly men and women benefit equally from prolonged resistance-type exercise training. J Gerontol A Biol Sci Med Sci 2013, 68, (7), 769-79.

19. Gorissen, S. H.; Burd, N. A.; Hamer, H. M.; Gijsen, A. P.; Groen, B. B.; van Loon, L. J., Carbohydrate coingestion delays dietary protein digestion and absorption but does not modulate postprandial muscle protein accretion. J Clin Endocrinol Metab 2014, 99, (6), 22508 .

20. Fried, L. P.; Tangen, C. M.; Walston, J.; Newman, A. B.; Hirsch, C.; Gottdiener, J.; Seeman, T.; Tracy, R.; Kop, W. J.; Burke, G.; McBurnie, M. A., Frailty in Older Adults: Evidence for a Phenotype. The Journals of Gerontology Series A: Biological Sciences and Medical Sciences 2001, 56, (3), M146-M157.

21. Leenders, M.; Verdijk, L. B.; Van der Hoeven, L.; Van Kranenburg, J.; Nilwik, R.; Wodzig, W. K.; Senden, J. M.; Keizer, H. A.; Van Loon, L. J., Protein supplementation during resistance-type exercise training in the elderly. Med Sci Sports Exerc 2013, 45, (3), $542-52$.

22. Noga, M. J.; Dane, A.; Shi, S.; Attali, A.; van Aken, H.; Suidgeest, E.; Tuinstra, T.; Muilwijk, B.; Coulier, L.; Luider, T.; Reijmers, T. H.; Vreeken, R. J.; Hankemeier, T., Metabolomics of cerebrospinal fluid reveals changes in the central nervous system metabolism in a rat model of multiple sclerosis. Metabolomics 2012, 8, (2), 253-263.

23. van der Kloet, F. M.; Bobeldijk, I.; Verheij, E. R.; Jellema, R. H., Analytical Error Reduction Using Single Point Calibration for Accurate and Precise Metabolomic Phenotyping. Journal of Proteome Research 2009, 8, (11), 5132-5141.

24. Mihaleva, V. V.; Korhonen, S. P.; van Duynhoven, J.; Niemitz, M.; Vervoort, J.; Jacobs, D. M., Automated quantum mechanical total line shape fitting model for quantitative NMR-based profiling of human serum metabolites. Anal Bioanal Chem 2014, 406, (13), 3091-102.

25. Szasz, G., Reaction-rate method for gamma-glutamyltransferase activity in serum. Clin Chem 1976, 22, (12), 2051-5.

26. Wold, H., Path models with latent variables: The NIPALS approach. Acad. Press: 1975.

27. Lê Cao, K.-A.; González, I.; Déjean, S., integrOmics: an R package to unravel relationships between two omics datasets. Bioinformatics 2009, 25, (21), 2855-2856.

28. Sébastien Lê, J. J., François Husson, FactoMineR: An R Package for Multivariate Analysis. Journal of Statistical Software 2008.

29. Smilde, A. K.; Jansen, J. J.; Hoefsloot, H. C. J.; Lamers, R.-J. A. N.; van der Greef, J.; Timmerman, M. E., ANOVA-simultaneous component analysis (ASCA): a new tool for analyzing designed metabolomics data. Bioinformatics 2005, 21, (13), 3043-3048.

30. Berger, A.; Milgram, E.; Mitchell, M.; Lawton, K.; Hanson, R.; Kalhan, S.; Milburn, M., The metabolomics of aging. Faseb Journal 2007, 21, (6), A1040-A1040. 
31. Lawton, K. A.; Berger, A.; Mitchell, M.; Milgram, K. E.; Evans, A. M.; Guo, L.; Hanson, R. W.; Kalhan, S. C.; Ryals, J. A.; Milburn, M. V., Analysis of the adult human plasma metabolome. Pharmacogenomics 2008, 9, (4), 383-397.

32. Laferrere, B.; Reilly, D.; Arias, S.; Swerdlow, N.; Gorroochurn, P.; Bawa, B.; Bose, M.; Teixeira, J.; Stevens, R. D.; Wenner, B. R.; Bain, J. R.; Muehlbauer, M. J.; Haqq, A.; Lien, L.; Shah, S. H.; Svetkey, L. P.; Newgard, C. B., Differential metabolic impact of gastric bypass surgery versus dietary intervention in obese diabetic subjects despite identical weight loss. Sci Transl Med 2011, 3, (80), 80re2.

33. Saccenti, E.; Hoefsloot, H. J.; Smilde, A.; Westerhuis, J.; Hendriks, M. W. B., Reflections on univariate and multivariate analysis of metabolomics data. Metabolomics 2014, 10, (3), 361-374.

34. Balagopal, P.; Rooyackers, O. E.; Adey, D. B.; Ades, P. A.; Nair, K. S., Effects of aging on in vivo synthesis of skeletal muscle myosin heavy-chain and sarcoplasmic protein in humans. Am J Physiol 1997, 273, (4 Pt 1), E790-800.

35. Short, K. R.; Vittone, J. L.; Bigelow, M. L.; Proctor, D. N.; Nair, K. S., Age and aerobic exercise training effects on whole body and muscle protein metabolism. Am J Physiol Endocrinol Metab 2004, 286, (1), E92-101.

36. Yarasheski, K. E.; Welle, S.; Nair, K. S., Muscle protein synthesis in younger and older men. JAMA 2002, 287, (3), 317-8.

37. Yarasheski, K. E.; Zachwieja, J. J.; Bier, D. M., Acute effects of resistance exercise on muscle protein synthesis rate in young and elderly men and women. Am J Physiol 1993, 265, (2 Pt 1), E210-4.

38. Rooyackers, O. E.; Adey, D. B.; Ades, P. A.; Nair, K. S., Effect of age on in vivo rates of mitochondrial protein synthesis in human skeletal muscle. Proc Natl Acad Sci U S A 1996, 93, (26), 15364-9.

39. Kjaer, M., Role of extracellular matrix in adaptation of tendon and skeletal muscle to mechanical loading. Physiol Rev 2004, 84, (2), 649-98.

40. Gosselin, L. E., Skeletal Muscle Collagen: Age, Injury and Disease. In SarcopeniaAge-Related Muscle Wasting and Weakness, Springer: 2011; pp 159-172.

41. Morris, S. M., Jr., Arginine metabolism: boundaries of our knowledge. J Nutr 2007, 137, (6 Suppl 2), 1602s-1609s.

42. Roberts, L. D.; Bostrom, P.; O'Sullivan, J. F.; Schinzel, R. T.; Lewis, G. D.; Dejam, A.; Lee, Y. K.; Palma, M. J.; Calhoun, S.; Georgiadi, A.; Chen, M. H.; Ramachandran, V. S.; Larson, M. G.; Bouchard, C.; Rankinen, T.; Souza, A. L.; Clish, C. B.; Wang, T. J.; Estall, J. L.; Soukas, A. A.; Cowan, C. A.; Spiegelman, B. M.; Gerszten, R. E., beta-Aminoisobutyric acid induces browning of white fat and hepatic beta-oxidation and is inversely correlated with cardiometabolic risk factors. Cell Metab 2014, 19, (1), 96-108.

43. Gomes, A. P.; Price, N. L.; Ling, A. J.; Moslehi, J. J.; Montgomery, M. K.; Rajman, L.; White, J. P.; Teodoro, J. S.; Wrann, C. D.; Hubbard, B. P.; Mercken, E. M.; Palmeira, C. M.; de Cabo, R.; Rolo, A. P.; Turner, N.; Bell, E. L.; Sinclair, D. A., Declining NAD(+) induces a pseudohypoxic state disrupting nuclear-mitochondrial communication during aging. Cell 2013, 155, (7), 1624-38.

44. Imai, S.; Guarente, L., NAD+ and sirtuins in aging and disease. Trends Cell Biol 2014, 24, (8), 464-71.

45. Overmyer, K. A.; Evans, C. R.; Qi, N. R.; Minogue, C. E.; Carson, J. J.; ChermsideScabbo, C. J.; Koch, L. G.; Britton, S. L.; Pagliarini, D. J.; Coon, J. J.; Burant, C. F., Maximal oxidative capacity during exercise is associated with skeletal muscle fuel selection and dynamic changes in mitochondrial protein acetylation. Cell Metab 2015, 21, (3), 468-78.

46. Pegg, A. E.; Mccann, P. P., Polyamine Metabolism and Function. American Journal of Physiology 1982, 243, (5), C212-C221. 
47. Turchanowa, L.; Rogozkin, V. A.; Milovic, V.; Feldkoren, B. I.; Caspary, W. F.; Stein, J., Influence of physical exercise on polyamine synthesis in the rat skeletal muscle. European Journal of Clinical Investigation 2000, 30, (1), 72-78.

48. Lee, N. K. L.; Maclean, H. E., Polyamines, Androgens, and Skeletal Muscle Hypertrophy. Journal of Cellular Physiology 2011, 226, (6), 1453-1460.

49. Kaminska, A. M.; Stern, L. Z.; Russell, D. H., Altered Muscle Polyamine Levels in Human Neuromuscular Diseases. Annals of Neurology 1981, 9, (6), 605-607.

50. Kaminska, A. M.; Stern, L. Z.; Russell, D. H., Polyamine Accumulation in Normal and Denervated Neonatal Muscle. Experimental Neurology 1981, 72, (3), 612-618.

51. Bongers, K. S.; Fox, D. K.; Kunkel, S. D.; Stebounova, L. V.; Murry, D. J.; Pufall, M. A.; Ebert, S. M.; Dyle, M. C.; Bullard, S. A.; Dierdorff, J. M.; Adams, C. M., Spermine oxidase maintains basal skeletal muscle gene expression and fiber size and is strongly repressed by conditions that cause skeletal muscle atrophy. Am J Physiol Endocrinol Metab 2015, 308, (2), E144-58.

52. Ost, M.; Keipert, S.; van Schothorst, E. M.; Donner, V.; van der Stelt, I.; Kipp, A. P.; Petzke, K. J.; Jove, M.; Pamplona, R.; Portero-Otin, M.; Keijer, J.; Klaus, S., Muscle mitohormesis promotes cellular survival via serine/glycine pathway flux. Faseb j 2015, 29, (4), 1314-28.

53. Sale, C.; Artioli, G. G.; Gualano, B.; Saunders, B.; Hobson, R. M.; Harris, R. C., Carnosine: from exercise performance to health. Amino Acids 2013, 44, (6), 1477-91.

54. Boldyrev, A. A.; Aldini, G.; Derave, W., Physiology and pathophysiology of carnosine. Physiol Rev 2013, 93, (4), 1803-45.

55. Harris, R. C.; Wise, J. A.; Price, K. A.; Kim, H. J.; Kim, C. K.; Sale, C., Determinants of muscle carnosine content. Amino Acids 2012, 43, (1), 5-12.

56. Kalish, B. T.; Fallon, E. M.; Puder, M., A tutorial on fatty acid biology. JPEN J Parenter Enteral Nutr 2012, 36, (4), 380-8.

57. Shimomura, Y.; Murakami, T.; Nakai, N.; Nagasaki, M.; Harris, R. A., Exercise promotes BCAA catabolism: effects of BCAA supplementation on skeletal muscle during exercise. J Nutr 2004, 134, (6 Suppl), 1583s-1587s.

58. Um, S. H.; D'Alessio, D.; Thomas, G., Nutrient overload, insulin resistance, and ribosomal protein S6 kinase 1, S6K1. Cell Metab 2006, 3, (6), 393-402.

59. Newgard, C. B., Interplay between lipids and branched-chain amino acids in development of insulin resistance. Cell Metab 2012, 15, (5), 606-14.

60. Schooneman, M. G.; Achterkamp, N.; Argmann, C. A.; Soeters, M. R.; Houten, S. M., Plasma acylcarnitines inadequately reflect tissue acylcarnitine metabolism. Biochim Biophys Acta 2014, 1841, (7), 987-94.

61. Herman, M. A.; She, P.; Peroni, O. D.; Lynch, C. J.; Kahn, B. B., Adipose tissue branched chain amino acid (BCAA) metabolism modulates circulating BCAA levels. $J$ Biol Chem 2010, 285, (15), 11348-56.

62. Brosnan, J. T., Glutamate, at the Interface between Amino Acid and Carbohydrate Metabolism. The Journal of Nutrition 2000, 130, (4), 988.

63. van de Poll, M. C.; Siroen, M. P.; van Leeuwen, P. A.; Soeters, P. B.; Melis, G. C.; Boelens, P. G.; Deutz, N. E.; Dejong, C. H., Interorgan amino acid exchange in humans: consequences for arginine and citrulline metabolism. The American Journal of Clinical Nutrition 2007, 85, (1), 167-172. 
1

2

3

4

5

6

7

8

9

10

11

12

13

14

15

16

17

18

19

20

21

22

23

24

25

26

27

28

29

30

31

32

33

34

35

36

37

38

39

40

41

42

43

44

45

46

47

48

49

50

51

52

53

54

55

56

57

58

59

60

ACS Paragon Plus Environment 


\section{TABLES}

Table 1.Characteristics of subjects of which skeletal muscle tissue biopsies were studied

\begin{tabular}{llll}
\hline & Young & Healthy older & Frail older \\
\hline $\mathrm{N}$ (male / female) & $30 / 0$ & $47 / 19$ & $25 / 18$ \\
Age (years) & $21.7 \pm 2.5$ & $71.7 \pm 5.2$ & $77.5 \pm 8.0$ \\
Height $(\mathrm{m})$ & $1.83 \pm 0.06$ & $1.72 \pm 0.08$ & $1.67 \pm 0.09$ \\
Weight $(\mathrm{kg})$ & $76.7 \pm 11.8$ & $75.9 \pm 13.3$ & $77.5 \pm 11.1$ \\
$\mathrm{BMI}^{\mathrm{a}}\left(\mathrm{kg} / \mathrm{m}^{2}\right)$ & $22.6 \pm 2.7$ & $25.5 \pm 3.0$ & $27.5 \pm 3.7$ \\
Body Fat $(\%)$ & $14.9 \pm 4.9$ & $24.5 \pm 5.6$ & $32.1 \pm 8.8$ \\
\hline Data was presented as mean \pm SD. a: body mass index, & &
\end{tabular}


Table 2. Muscle metabolites that are significantly different between healthy older and young males ${ }^{\text {a }}$

\begin{tabular}{|c|c|c|}
\hline Metabolite & $P$-value & $\begin{array}{l}\mathrm{FC}^{\mathrm{b}} \\
\text { (Older/Young) }\end{array}$ \\
\hline \multicolumn{3}{|l|}{ TCA Cycle } \\
\hline Succinic acid & 0.02 & 0.76 \\
\hline 2-ketoglutaric acid & 0.03 & 0.76 \\
\hline Fumaric acid & 0.04 & 0.82 \\
\hline Lactic acid & 0.05 & 0.69 \\
\hline \multicolumn{3}{|l|}{ Energy } \\
\hline ATP & $<0.01$ & 0.75 \\
\hline $\mathrm{ADP}$ & 0.01 & 0.88 \\
\hline \multicolumn{3}{|l|}{ Branched chain amino acids } \\
\hline Valine & $<0.01$ & 0.81 \\
\hline Leucine & $<0.01$ & 0.81 \\
\hline Isoleucine & 0.03 & 0.84 \\
\hline \multicolumn{3}{|l|}{ Acylcarnitines } \\
\hline Acetylcarnitine (C2) & $<0.01$ & 0.49 \\
\hline Malonylcarnitine (C3-DC) & $<0.01$ & 0.46 \\
\hline \multicolumn{3}{|l|}{ Intracellular buffering } \\
\hline Carnosine & $<0.01$ & 0.7 \\
\hline \multicolumn{3}{|l|}{ Arginine, Proline Pathway } \\
\hline Ornithine & $<0.01$ & 1.55 \\
\hline Arginine & $<0.01$ & 1.34 \\
\hline 4-hydroxy-proline & 0.01 & 0.69 \\
\hline Proline & 0.02 & 0.84 \\
\hline Glycylglycine & 0.05 & 0.87 \\
\hline Methionine & $<0.01$ & 0.8 \\
\hline \multicolumn{3}{|l|}{ Other amino acids } \\
\hline Lysine & $<0.01$ & 1.44 \\
\hline Aspartic acid & $<0.01$ & 1.45 \\
\hline \multicolumn{3}{|l|}{ Oxylipins } \\
\hline \multicolumn{3}{|l|}{ LA (LOX) } \\
\hline 9-HODE & $<0.01$ & 1.4 \\
\hline 13-HODE & 0.01 & 1.37 \\
\hline 13-KODE & 0.03 & 1.3 \\
\hline \multicolumn{3}{|l|}{ LA(CYP450) } \\
\hline 9,10-EpOME & 0.04 & 1.39 \\
\hline \multicolumn{3}{|l|}{ ALA (LOX) } \\
\hline 9-HOTrE & 0.01 & 1.71 \\
\hline \multicolumn{3}{|l|}{ DGLA (LOX) } \\
\hline 15S-HETrE & 0.02 & 0.83 \\
\hline 8-HETrE & 0.03 & 0.85 \\
\hline
\end{tabular}


1

2

3

4

5

6

7

8

9

10

11

12

13

14

15

16

17

18

19

20

21

22

23

24

25

26

27

28

29

30

31

32

33

34

35

36

37

38

39

40

41

42

43

44

45

46

47

48

49

50

51

52

53

54

55

56

57

58

59

60 
1

2

3

4

5

6

Table 3.Muscle metabolites that are significantly different between frail and healthy older subjects. ${ }^{\text {a }}$

\begin{tabular}{|c|c|c|c|c|}
\hline Metabolite & Group & Sex & Interaction & FC $^{\text {b }}$ (Frail/Healthy) \\
\hline \multicolumn{5}{|l|}{ TCA Cycle } \\
\hline Citric acid & $<0.01$ & $\mathrm{NS}^{\mathrm{c}}$ & NS & 0.54 \\
\hline \multicolumn{5}{|l|}{ Acylcarnitines } \\
\hline Isovalerylcarnitine (C5) & $<0.01$ & NS & NS & 0.42 \\
\hline Octenoylcarnitine (C8) & 0.03 & 0.03 & NS & 0.77 \\
\hline Malonylcarnitine(C3-DC) & 0.02 & NS & NS & 0.77 \\
\hline Carnitine $(\mathrm{C} 0)$ & 0.01 & NS & NS & 0.75 \\
\hline \multicolumn{5}{|l|}{ Intracellular buffering } \\
\hline Carnosine & 0.01 & NS & NS & 0.8 \\
\hline \multicolumn{5}{|l|}{ Oxylipins } \\
\hline \multicolumn{5}{|l|}{ LA (CYP450) } \\
\hline 12,13DiHOME & 0.04 & NS & 0.03 & 1.18 \\
\hline \multicolumn{5}{|l|}{ DGLA (LOX) } \\
\hline 8HETrE & 0.03 & NS & NS & 0.81 \\
\hline 15SHETrE & $<0.01$ & NS & NS & 0.77 \\
\hline \multicolumn{5}{|l|}{ Polyamine metabolism } \\
\hline Spermidine & 0.01 & 0.02 & NS & 1.24 \\
\hline Spermine & 0.04 & NS & NS & 0.9 \\
\hline \multicolumn{5}{|l|}{ Other amino acids } \\
\hline Histidine & $<0.01$ & NS & NS & 0.79 \\
\hline Asparagine & 0.01 & NS & NS & 0.81 \\
\hline Taurine & 0.01 & NS & NS & 0.79 \\
\hline Serine & 0.01 & NS & NS & 0.86 \\
\hline Glycine & 0.02 & NS & NS & 0.81 \\
\hline oacetylserine & 0.02 & NS & NS & 0.9 \\
\hline Homoserine & 0.02 & NS & NS & 0.85 \\
\hline Tyrosine & 0.02 & NS & NS & 0.83 \\
\hline Tryptophan & 0.02 & 0.04 & NS & 0.83 \\
\hline Methionine & 0.02 & NS & NS & 0.83 \\
\hline Glutamine & 0.03 & NS & NS & 0.82 \\
\hline Pyroglutamic acid & 0.03 & NS & NS & 0.83 \\
\hline
\end{tabular}


2

3

4

5

6

7

8

9

a: Metabolites are presented that significantly $(P$-value $=<0.05)$ differ between frail and healthy older subjects according to a univariate ANOVA models that accounted for Group effect (Frail vs. Healthy), Sex effect and Group-Sex interaction; Significance of Group (Frail vs. Healthy older subjects) effects and Group-Sex interactions has been indicated; $b$ :fold change; c: not significant 
Table 4. Training effect on muscle metabolites in healthy older subjects ${ }^{a}$

\begin{tabular}{|c|c|c|}
\hline Metabolite & Training & FC(post/pre training) ${ }^{b}$ \\
\hline Pipecolic acid & 0.002 & 1.64 \\
\hline Isovalerylcarnitine (C5) & 0.005 & 0.56 \\
\hline Linoleylcarnitine (C18:2) & 0.01 & 0.61 \\
\hline Oleylcarnitine (C18:1) & 0.01 & 0.7 \\
\hline Propionylcarnitine (C3) & 0.01 & 0.73 \\
\hline Palmitoylcarnitine (C16) & 0.02 & 0.75 \\
\hline 11.12.EpETrE & 0.03 & 1.26 \\
\hline Tetradecenoylcarnitine (C14:1 & 0.03 & 0.47 \\
\hline $\mathrm{AAAC}^{\mathrm{c}}$ & 0.02 & 0.77 \\
\hline FAAC $^{\mathrm{d}}$ & NS & 0.96 \\
\hline \multicolumn{3}{|c|}{$\begin{array}{l}\text { a: Metabolites are presented that significantly }(P \text {-value }=<0.05) \text { differ pre- and } \\
\text { post-training in healthy older subjects according to univariate linear mixed models. } \\
\text { We note that we constructed also linear mixed models that account for other factors } \\
\text { and their interactions, but these were not found to be significant b: fold change, } \\
\text { c,d: Single Value Decomposition (SVD) were calculated for amino acid and fatty acid } \\
\text { acylcarnitines, denoted as AAAC and FAAC, respectively. }\end{array}$} \\
\hline
\end{tabular}


Table 5. Training effect on muscle metabolites in frail older subjects ${ }^{\text {a }}$

\begin{tabular}{llllll}
\hline Metabolite & Training & Supplement & Sex & Interaction & FC(post/pre training) $^{\mathrm{b}}$ \\
\hline Propionylcarnitine (C3) & $<0.01$ & $\mathrm{NS}^{\mathrm{c}}$ & $\mathrm{NS}$ & $\mathrm{NS}$ & 0.75 \\
Glucose & $<0.01$ & $\mathrm{NS}$ & $<0.01$ & $\mathrm{NS}$ & 1.35 \\
Lactic acid & 0.01 & $\mathrm{NS}$ & $<0.01$ & $\mathrm{NS}$ & 1.55 \\
Tetradecenoylcarnitine (C14:1) & 0.03 & $\mathrm{NS}$ & $\mathrm{NS}$ & $\mathrm{NS}$ & 2.00 \\
Methionine & 0.04 & $<0.01$ & $\mathrm{NS}$ & $\mathrm{NS}$ & 1.22 \\
Tryptophan & 0.04 & $\mathrm{NS}$ & $\mathrm{NS}$ & $\mathrm{NS}$ & 1.21 \\
$\beta$ Alanine & 0.05 & $\mathrm{NS}$ & $\mathrm{NS}$ & 0.01 & 0.92 \\
Isoleucine & 0.05 & $<0.01$ & 0.02 & $\mathrm{NS}$ & 1.21 \\
Myristoylcarnitine (C14) $^{\mathrm{N}}$ & 0.05 & $\mathrm{NS}$ & $\mathrm{NS}$ & $\mathrm{NS}$ & 1.73 \\
AAAC $^{\mathrm{d}}$ & 0.01 & $\mathrm{NS}$ & $\mathrm{NS}$ & $\mathrm{NS}$ & 0.76 \\
FAAC $^{\mathrm{e}}$ & $\mathrm{NS}$ & $\mathrm{NS}$ & $\mathrm{NS}$ & $\mathrm{NS}$ & 1.95 \\
\hline
\end{tabular}

a: Metabolites are presented that significantly $(P$-value $=<0.05)$ differ pre- and post-training in frail older subjects according to univariate linear mixed models that account for Supplement, Sex and (Training and Supplement) Interaction. We note that also linear mixed models were constructed that account for other interactions, but these were not found to be significant; b: fold change, c: Not Significant, d \& e: Single Value Decomposition (SVD) were calculated for amino acid and fatty acid acylcarnitines (AAAC and FAAC, respectively). 


\section{FIGURE LEGENDS}

Figure 1. Principal component analysis (PCA) plot of biogenic amines detected in muscle biopsies. To visualize whether groups are significantly different from each other, confidence ellipses (95\% Confidence Interval) were drawn around them.

Figure 2. Correlation network of muscle metabolites and genes. Only significantly changed genes were selected $(\mathrm{FDR}<0.05)$. Metabolite canonical correlation cutoff $<=0.80$. Circle: gene, rectangle: metabolite, Green : positive correlation.

Figure 3. Pearson correlation heatmap of muscle to muscle metabolites (a), plasma to plasma metabolites (b), muscle to plasma metabolites (c). (d) Pearson correlation network of the most strongly correlated muscle and plasma metabolites. Red and blue indicate positive and negative correlations, respectively. Thick lines: correlation $\sim 0.5$, thin lines: $0.3<$ correlation $<0.5$. Pink nodes: muscle tissue (T) metabolites and yellow nodes: plasma (P) metabolites,

Figure 4. (a) Schematic representation of mitochondrial oxidation of free fatty acids (FFA) and branched chain amino acids (BCAA). Arrows indicate effect of prolonged resistance-type exercise training on older subjects (healthy and frail) on BCAA (increase) and acylcarnitines (decrease) as well as the proposed (dashed-arrows) downregulation of the branched chain $\alpha$ keto acid hydrogenase (BCKDH) complex. (b) Schematic representation of age-related $\mathrm{NAD}^{+}$ dependent acetylation of PGC1 $\alpha$. Dashed arrows indicate $\mathrm{NAD}^{+}$and SIRT1 dependent downregulation of PGC- $1 \alpha$ and $\beta$-isoamino butyric acid (BAIBA). 
Figure 1.

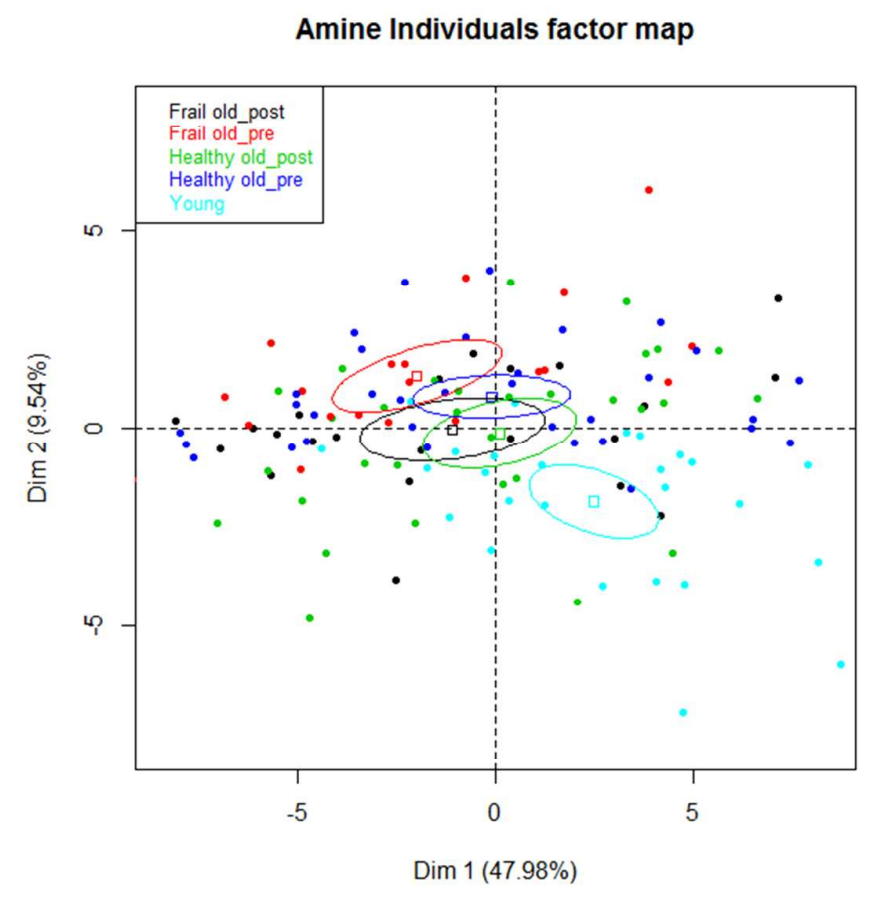

ACS Paragon Plus Environment 


\section{Figure 2.}

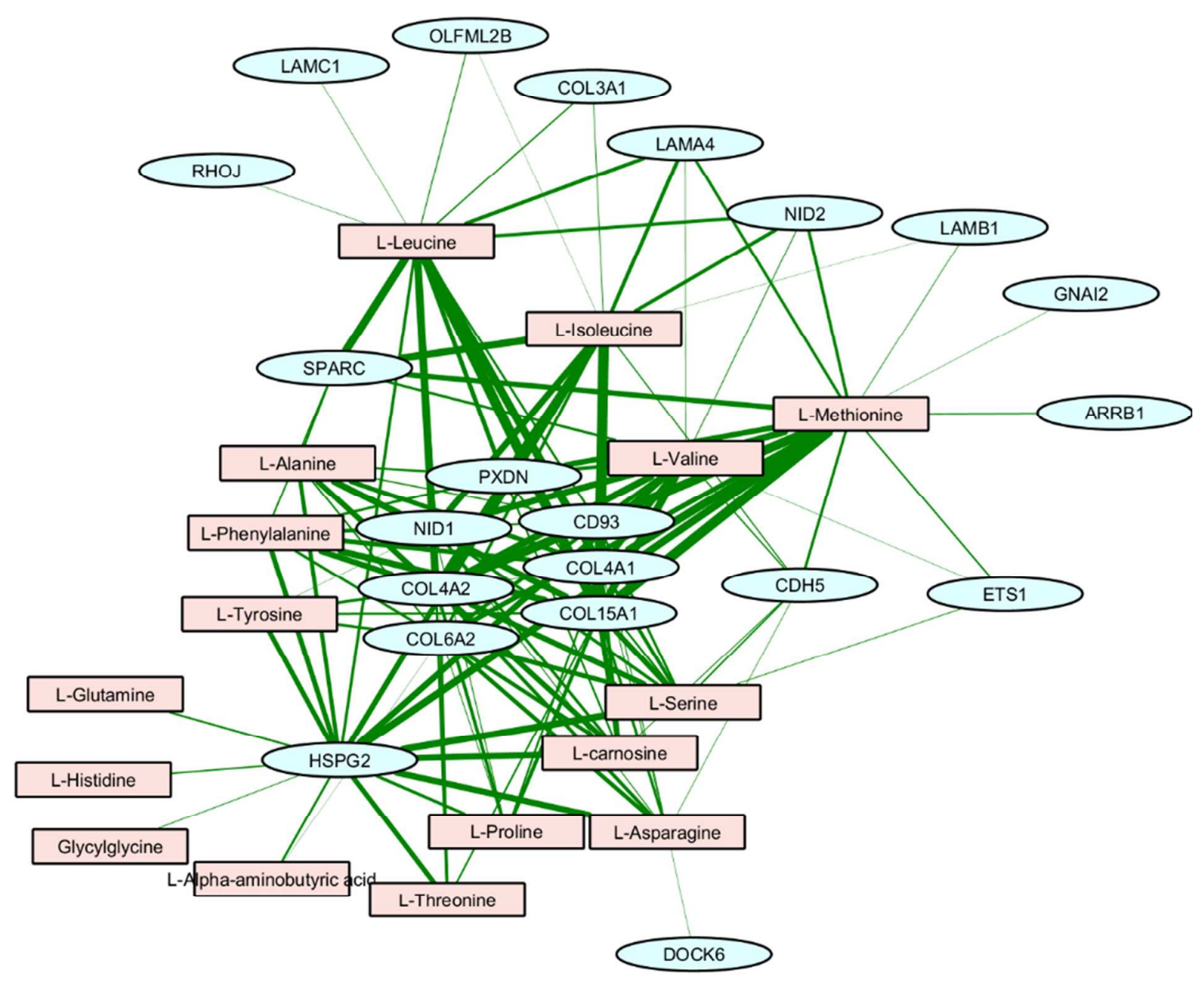


Figure 3.

(a)

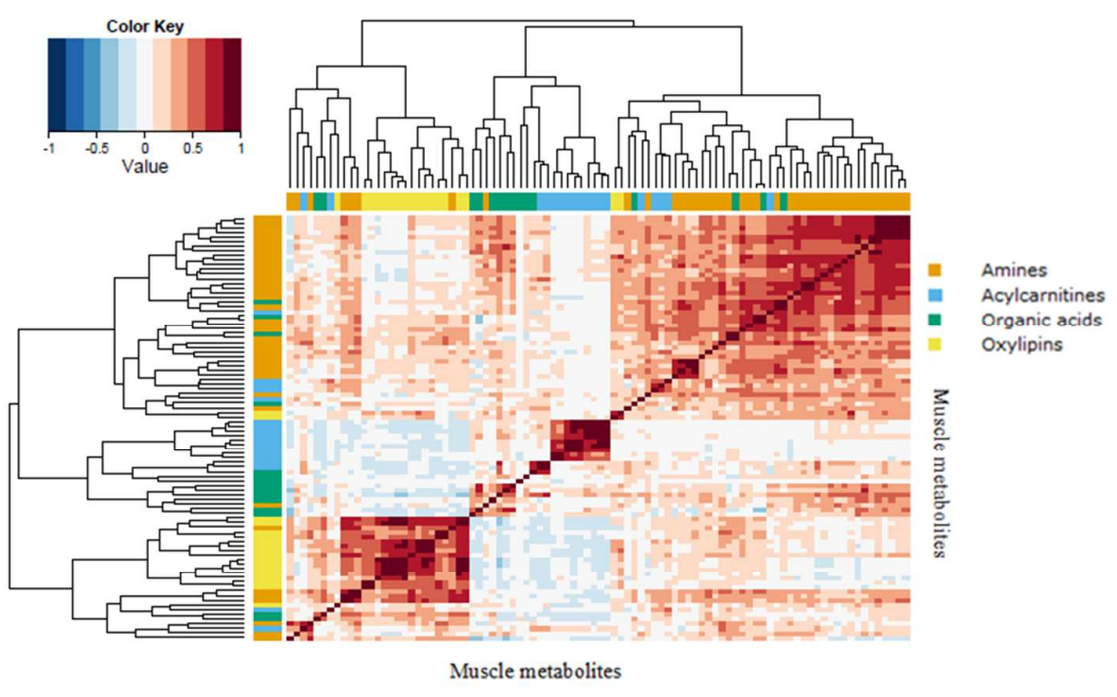

(b)

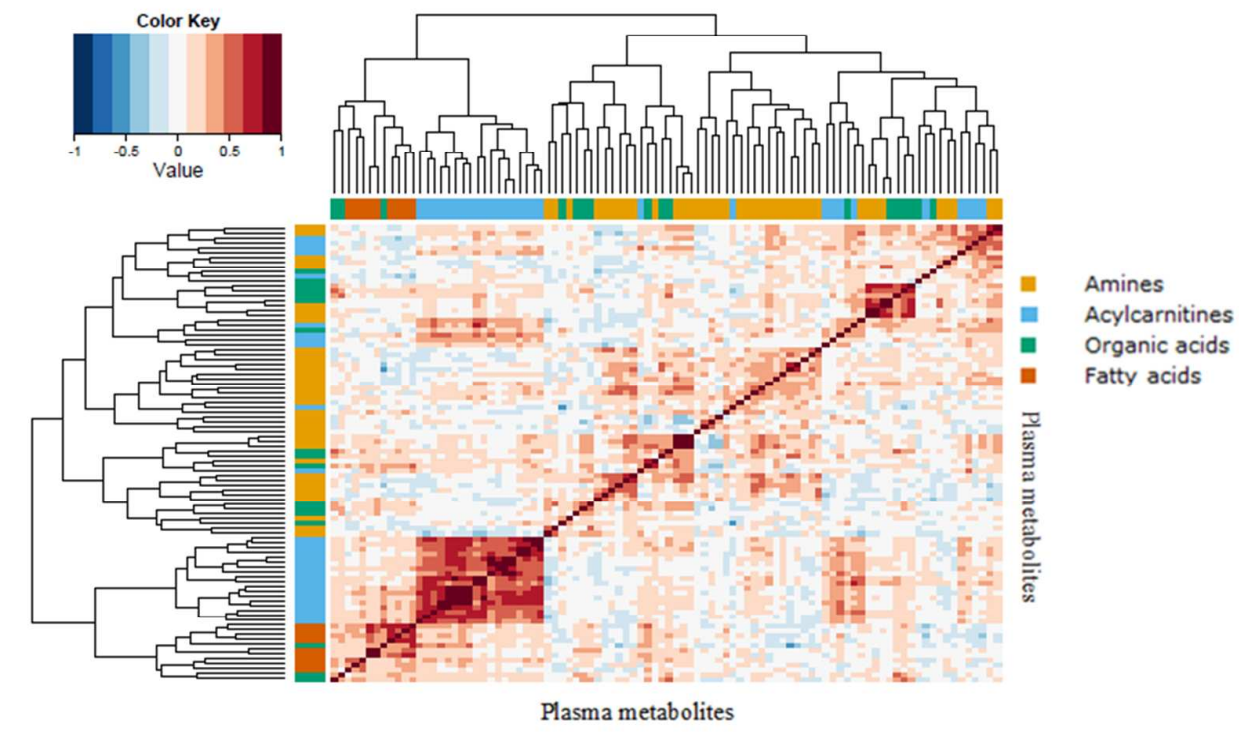




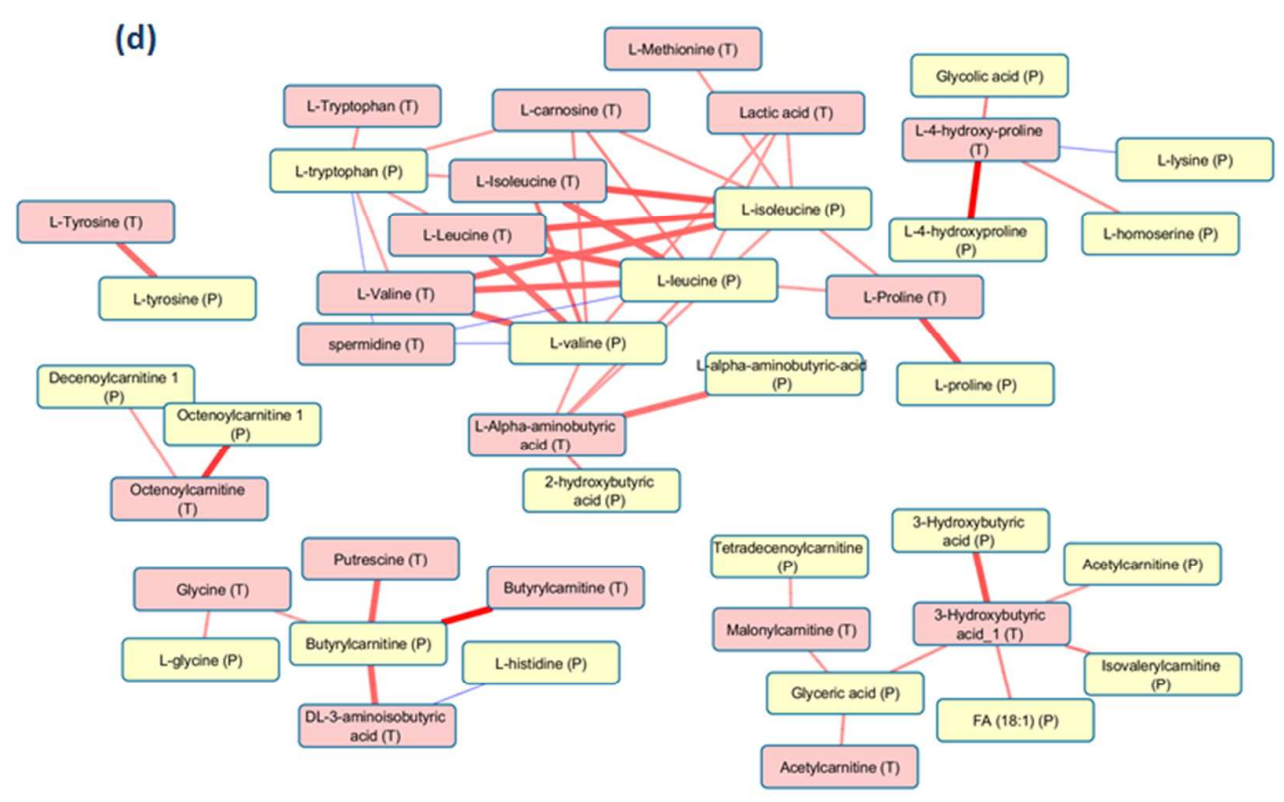


Figure 4.
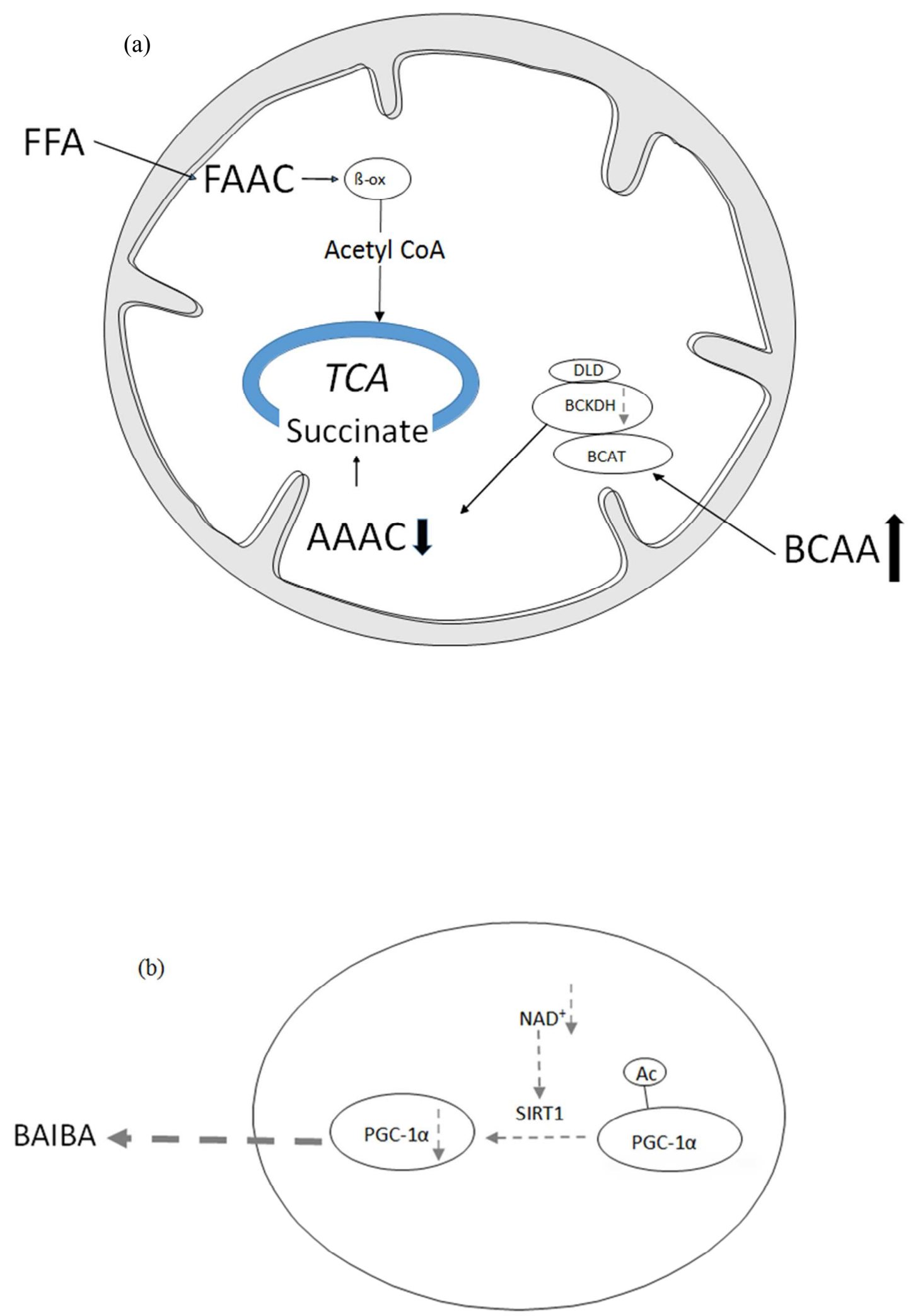

ACS Paragon Plus Environment 
1

2

3

4

5

6

7

8

9

10

11

12

13

14

15

16

17

18

19

20

21

22

23

24

25

26

27

28

29

30

31

32

33

34

35

36

37

38

39

40

41

42

43

44

45

46

47

48

49

50

51

52

53

54

55

56

57

58

59

60

ACS Paragon Plus Environment 


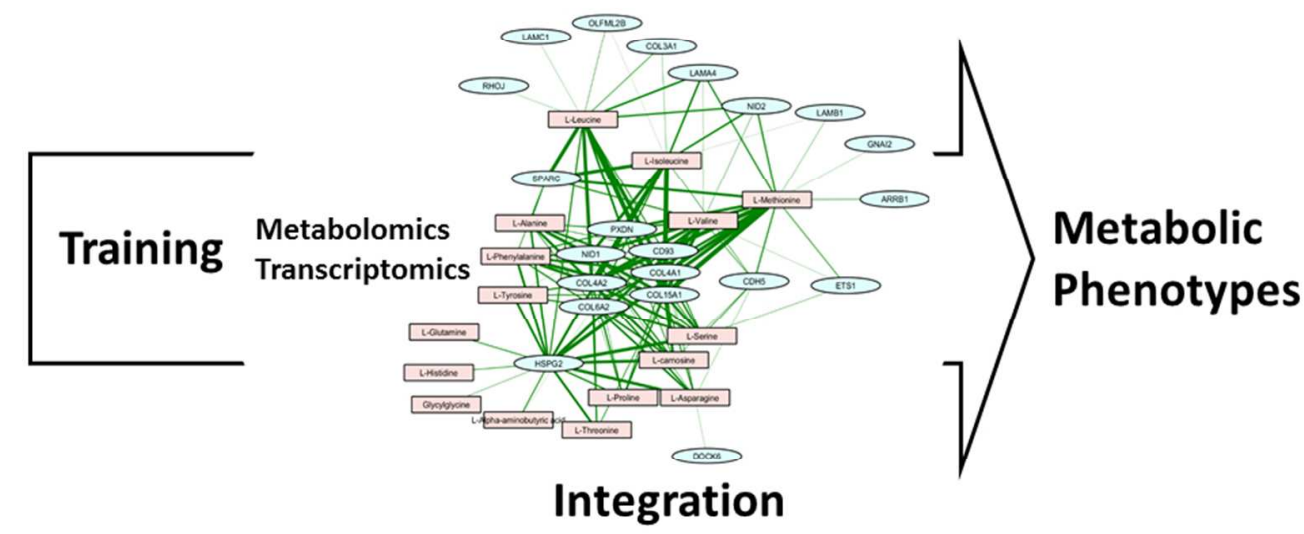

For TOC only

254×190mm (96 x 96 DPI) 